\title{
Sex-related differences in sleep slow wave activity in major depressive disorder: a high-density EEG investigation
}

\author{
David T Plante*, Eric C Landsness, Michael J Peterson, Michael R Goldstein, Brady A Riedner, Timothy Wanger, \\ Jeffrey J Guokas, Giulio Tononi and Ruth M Benca
}

\begin{abstract}
Background: Sleep disturbance plays an important role in major depressive disorder (MDD). Prior investigations have demonstrated that slow wave activity (SWA) during sleep is altered in MDD; however, results have not been consistent across studies, which may be due in part to sex-related differences in SWA and/or limited spatial resolution of spectral analyses. This study sought to characterize SWA in MDD utilizing high-density electroencephalography (hdEEG) to examine the topography of SWA across the cortex in MDD, as well as sex-related variation in SWA topography in the disorder.

Methods: All-night recordings with 256 channel hdEEG were collected in 30 unipolar MDD subjects (19 women) and 30 age and sex-matched control subjects. Spectral analyses of SWA were performed to determine group differences. SWA was compared between MDD and controls, including analyses stratified by sex, using statistical non-parametric mapping to correct for multiple comparisons of topographic data.

Results: As a group, MDD subjects demonstrated significant increases in all-night SWA primarily in bilateral prefrontal channels. When stratified by sex, MDD women demonstrated global increases in SWA relative to age-matched controls that were most consistent in bilateral prefrontal regions; however, MDD men showed no significant differences relative to age-matched controls. Further analyses demonstrated increased SWA in MDD women was most prominent in the first portion of the night.
\end{abstract}

Conclusions: Women, but not men with MDD demonstrate significant increases in SWA in multiple cortical areas relative to control subjects. Further research is warranted to investigate the role of SWA in MDD, and to clarify how increased SWA in women with MDD is related to the pathophysiology of the disorder.

\section{Background}

Sleep disturbance has diagnostic, clinical, and functional significance in major depressive disorder (MDD). Selfreport of insomnia and/or hypersomnia is a diagnostic criterion for MDD, and sleep-related complaints occur in the majority of MDD patients [1,2]. Sleep-related clinical complaints are important in the natural history of the disorder, as they increase the risk of developing a depressive episode [3-7], attempting suicide [8,9], and relapsing after remission [10-12]. Additionally, myriad studies have examined polysomnographic measures in

\footnotetext{
* Correspondence: dplante@wisc.edu

Department of Psychiatry, University of Wisconsin School of Medicine and Public Health, Madison, WI, USA
}

MDD, demonstrating alterations in sleep continuity, rapid eye movement (REM) sleep, and slow wave sleep (SWS) $[13,14]$. Scoring of SWS relies on visual assessment of frequency, amplitude, and proportion of delta waves $[15,16]$, which can occur in all stages of NREM sleep and are not exclusive to SWS. Slow wave activity (SWA), which represents the power density in the $1-4.5 \mathrm{~Hz}$ range in all stages of NREM sleep, more accurately captures the variation in slow oscillations during sleep that is not reflected by traditional sleep staging $[17,18]$.

SWA has been established as a marker of sleep homeostasis, as it increases in proportion to prior wakefulness and declines with sleep $[19,20]$. Based in part on the antidepressant effects of sleep deprivation, it has

\section{Biomed Central}


been hypothesized that MDD involves deficiency in sleep homeostatic processes-the S-deficiency hypothesis of depression [21,22]. Prior studies that have examined SWA in MDD using spectral analysis support this hypothesis, particularly suggesting decrements of SWA in the first portion of the night $[23,24]$. However, findings have not been consistent across studies, with other investigations failing to demonstrate significant differences in SWA in depressed subjects relative to healthy controls [25-28]. Moreover, significant sex-related effects on SWA in MDD have been described, with increases in SWA among depressed women and decreases in SWA among depressed men, relative both to each other and to healthy comparison subjects [29-31].

A major limitation of prior studies of SWA in MDD is the use of limited central derivations of the electroencephalogram (EEG) to quantify SWA. Since SWA is most prominent during sleep in frontal brain regions [32-35], the use of central channels to assess SWA may not optimally reflect differences in sleep homeostatic processes in depression. Furthermore, the use of a single or only a few EEG derivations to perform spectral analysis provides limited spatial resolution, and thus, topographic alterations in SWA would not be evident using these approaches. Therefore, this study was conducted using high-density (hd) EEG to evaluate the topography of sleep SWA in MDD relative to healthy age and sexmatched comparison subjects. We hypothesized that differences in SWA between MDD and control subjects would be most pronounced in frontal channels, and that there would be differential effects of sex on SWA in MDD subjects, with women with MDD demonstrating increases and men with MDD demonstrating reductions in SWA compared to age and sex-matched healthy comparison subjects.

\section{Methods \\ Subjects}

Thirty right-handed outpatient MDD subjects (19 female) were selected from a larger study on sleep homeostasis in neuropsychiatric disorders, conducted at the University of Wisconsin-Madison. MDD was diagnosed via the Structured Clinical Interview for DSM-IV Axis I disorders (SCID) [36] and global depression severity was evaluated with the clinician-administered 17-item Hamilton Rating Scale for Depression (HRSD) [37]. For inclusion, subjects were required to have at least moderate depression defined as HRSD > 15 and be free of psychotropic medications (or other agents that could alter sleep architecture) for $\geq 1$ month. In addition, subjects were unipolar and had no history of psychosis or active drug/ alcohol dependence. Subjects were free of significant neurological and medical conditions, including evidence of sleep disordered breathing and sleep related movement disorders, verified by in-laboratory polysomnography (see below). Age and sex-matched healthy comparison subjects were evaluated with the nonpatient SCID [38] to rule out current or past psychiatric disorders.

All subjects provided informed consent and were instructed to maintain regular sleep-wake schedules, avoid napping, and to limit the use of caffeinated or alcoholic beverages for the duration of the study. Adherence was monitored using sleep-diaries and wrist motor actigraphy (Actiwatch, Mini-Mitter, Bend, OR). This study was approved by the Institutional Review Board of the University of Wisconsin-Madison.

\section{Study design}

All subjects underwent in-laboratory hdEEG polysomnography (PSG) that utilized 256 channel hdEEG (Electrical Geodesics Inc., Eugene, OR), as well as standard monitoring with electrooculogram (EOG), sub-mental electromyogram (EMG), electrocardiogram (ECG), bilateral tibial EMG, respiratory inductance plethysmography, pulse oximetry, and a position sensor. Participants arrived at the laboratory between 20:00 and 21:00 for set-up that took approximately two hours, and then were allowed to sleep undisturbed in the laboratory beginning within one hour of their usual bedtime. Sleep hdEEG recordings were collected with vertex-referencing, using NetStation software (Electrical Geodesics Inc., Eugene, OR).

\section{Spectral analysis}

HdEEG signals were sampled at $500 \mathrm{~Hz}$, first-order high-pass filtered in NetStation $(0.1 \mathrm{~Hz})$, downsampled to $128 \mathrm{~Hz}$, band-pass filtered (2-way least-squares FIR, 1-40 Hz) in MATLAB (The MathWorks Inc., Natick, $\mathrm{MA}$ ), and average-referenced to the average scalp voltage computed in all channels. Semi-automatic artifact rejection was conducted to remove channels with highfrequency noise or interrupted contact with the scalp during individual epochs. Channels for which artifact affected the majority of the recording were excluded. Spectral analysis of NREM sleep was performed for each channel in consecutive 6-second epochs (Welch's averaged modified periodgram with a Hamming window). To increase the signal-to-noise ratio, analyses were restricted to the 185 channels overlaying the scalp [39]. Sleep staging was performed by a registered polysomnographic technologist in 30-second epochs according to standard criteria [16] using Alice ${ }^{\circledR}$ Sleepware (Philips Respironics, Murrysville, PA) based on 6 EEG channels at approximate 10-20 locations (F3, F4, C3, C4, O1, and O2) re-referenced to the mastoids, sub-mental EMG and EOG. 


\section{Slow wave activity time course}

SWA time course was quantified using similar methods described by other groups $[29,40]$. The exponential decay function utilized was as follows: $\mathrm{SWA}_{t}=\left(\mathrm{SWA}_{0} * \mathrm{e}^{-r t}\right)+$ $\mathrm{SWA}_{\infty}$, in which $\mathrm{SWA}_{t}$ is the SWA value at a given time point $t, \mathrm{SWA}_{0}$ represents the hypothetical SWA value at time $0, t$ indicates time ( $\mathrm{min}$ ) from sleep onset, $r$ is the rate $\left(\mathrm{min}^{-1}\right)$ of exponential decay, and $\mathrm{SWA}_{\infty}$ indicates the SWA value of the decay function's asymptote. To optimize fit for each decay function, for each subject, relative SWA for each NREM period was calculated as a percent of the average SWA across the first NREM periods 1-4, and NREM period midpoints were used to derive the corresponding time point values. NREM periods were defined using duration and endpoint criteria in a manner similar to prior studies [41], with the exception that NREM periods were inclusive of all stages of NREM sleep (N1-N3).

\section{Statistics}

Differences in all-night SWA (both global average of 185 channels and channel-by-channel) between MDD and matched controls were examined using 2-tailed, unpaired t-tests. Given aforementioned sex-related differences in SWA in MDD [29,30], as well as reported differences in SWA between healthy men and women that vary by age [42-44], additional analyses between MDD and controls stratified by sex were performed to maintain age-matching of subjects. Also, effects of NREM period were explored given the known decline of SWA across NREM periods and prior studies that have demonstrated decrements in SWA in MDD primarily occurring in the first NREM period [24]. To correct for multiple comparisons of the topographical hdEEG data, statistical non-parametric mapping with suprathreshold cluster tests was utilized [45]. For correlational analyses of SWA with polysomnographic and clinical data, hdEEG sleep data were first cleaned for outliers using a threshold of \pm 2.5 standard deviations from the mean at each channel. Statistical relationship between variables and SWA in each channel was assessed using linear regression. To limit spurious findings due to multiple comparisons, correlation analyses of PSG variables were limited to those measures significantly different between groups. Additionally, topographic correlations were considered significant only if clusters of $\geq 3$ contiguous channels were significant at $\alpha=0.05$ within cortical regions of significant difference between groups. Statistical analyses were performed using MATLAB (The MathWorks Inc., Natick, MA) and STATISTICA (StatSoft Inc., Tulsa, OK).

\section{Results}

MDD subjects were young to middle aged (mean $26.0 \pm$ 9.1; range 18 to 53 years) with moderate depression (mean HRSD $18.6 \pm 2.7$; range 15 to 25 ) and had not taken psychotropic medications within $\geq 5$ months of enrollment (Table 1). Eleven MDD participants reported at least one prior depressive episode. $\mathrm{HC}$ subjects did not report a personal or family history of mood disorders. Polysomnography demonstrated significant differences

Table 1 Demographic, clinical, and polysomnographic data

\begin{tabular}{|c|c|c|c|c|c|c|c|c|c|}
\hline & $\begin{array}{c}\text { MDD } \\
(N=30)\end{array}$ & $\frac{\mathrm{HC}}{(N=30)}$ & $p$ & $\frac{\text { MDD Females }}{(N=19)}$ & $\frac{\text { HC Females }}{(N=19)}$ & $p$ & $\frac{\text { MDD Males }}{(N=11)}$ & $\frac{\text { HC Males }}{(N=11)}$ & $p$ \\
\hline Age (years) & $26.0(9.1)$ & $25.4(8.5)$ & .793 & $23.4(5.6)$ & $23.1(6.2)$ & .891 & $30.6(12.1)$ & $29.4(10.7)$ & .811 \\
\hline HRSD-17 & $18.6(2.7)$ & - & & $19.0(3.0)$ & - & & $17.9(1.6)$ & - & \\
\hline TST (min.) & 377.8 (66.3) & $412.7(47.5)$ & .023 & $390.5(54.0)$ & $419.6(49.8)$ & .093 & $356.0(81.7)$ & $400.8(42.9)$ & .122 \\
\hline WASO (min.) & $43.3(30.2)$ & $30.2(20.7)$ & .055 & $38.1(26.4)$ & 30.7 (20.6) & .345 & $52.4(35.4)$ & $29.4(21.8)$ & .082 \\
\hline Al (\#/hr.) & $10.6(5.6)$ & $7.9(4.3)$ & .043 & $9.4(5.5)$ & $8.2(3.8)$ & .424 & $12.6(5.5)$ & $7.4(5.3)$ & .036 \\
\hline SE (\%) & $86.5(7.9)$ & $89.6(7.4)$ & .132 & $88.7(7.1)$ & $89.2(8.6)$ & .846 & $82.8(8.1)$ & $90.1(4.9)$ & .018 \\
\hline SOL (min.) & $15.5(16.5)$ & $18.6(24.3)$ & .566 & $11.6(10.5)$ & $20.6(28.0)$ & .199 & $22.1(22.7)$ & $15.1(16.7)$ & .414 \\
\hline N1 (\%) & $8.1(5.3)$ & $7.9(4.7)$ & .926 & $7.5(5.5)$ & $7.7(5.0)$ & .897 & $9.0(5.0)$ & $8.3(4.2)$ & .724 \\
\hline N2 (\%) & $57.7(8.1)$ & $60.4(6.1)$ & .151 & $56.6(8.6)$ & $60.4(5.2)$ & .111 & $59.5(7.2)$ & $60.4(7.8)$ & .788 \\
\hline N3 (\%) & $16.8(9.2)$ & $14.8(7.1)$ & .348 & $20.0(7.6)$ & $15.9(6.3)$ & .075 & $11.2(9.3)$ & $13.0(8.2)$ & .651 \\
\hline REM (\%) & $18.1(6.6)$ & $16.9(5.0)$ & .448 & $15.9(6.5)$ & $16.0(5.2)$ & .923 & $22.3(4.8)$ & $18.4(4.5)$ & .071 \\
\hline REML (min.) & $131.2(77.4)$ & $122.2(69.7)$ & .641 & $158.1(78.4)$ & $131.1(71.2)$ & .275 & $80.1(43.7)$ & $106.7(67.4)$ & .302 \\
\hline $\operatorname{SWA}\left(\mu \mathrm{V}^{2} / \mathrm{Hz}\right)$ & $22.7(13.1)$ & $17.3(6.8)$ & .054 & $28.8(12.4)$ & $19.3(6.8)$ & .006 & $12.1(5.4)$ & $13.9(5.3)$ & .442 \\
\hline
\end{tabular}

$M D D$, major depressive disorder; $H C$, healthy control; HRSD-17, Hamilton Rating Scale for Depression (17-item); TST, total sleep time; WASO, wake after sleep onset; $A l$, arousal index; SE, sleep efficiency (TST/time in bed); SOL, sleep onset latency; N1/2/3, NREM stage 1/2/3 (\% of TST); REM, stage REM (\% of TST); REML, REM latency (time from sleep onset to first REM sleep epoch); SWA, global slow wave activity (EEG power in $1-4.5 \mathrm{~Hz}$ range average 185 channels); Values are displayed as mean (standard deviation).

*2-tailed, independent samples $t$-tests. 
between groups, with MDD subjects demonstrating decreased total sleep time (TST) and increased arousal index (AI) (Table 1). When stratified by sex, there were no significant differences in polysomnographic variables between women with MDD and female controls, but depressed men had significantly higher AI and lower sleep efficiency (SE) than male controls (Table 1).

All-night global SWA (average of 185 channels) was not significantly different between MDD and $\mathrm{HC}$ groups; however, there was a trend towards increased global SWA in MDD (Table 1). Topographic analysis demonstrated significantly increased SWA in MDD relative to $\mathrm{HC}$, primarily in bilateral prefrontal channels, as well as left lateral parietal and occipital channels (Figure 1).

When groups were stratified by sex, female MDD subjects demonstrated significantly higher global all-night SWA compared to age-matched female controls (28.8 $\mu \mathrm{V}^{2} / \mathrm{Hz} \pm 12.4$ vs. $\left.19.3 \mu \mathrm{V}^{2} / \mathrm{Hz} \pm 6.8, \mathrm{p}=0.006\right)$. Topographic analysis demonstrated significant increases in SWA in MDD women relative to $\mathrm{HC}$ women that were most consistent in bilateral prefrontal channels, and additionally significant in multiple other cortical regions including frontal, lateral parietal, and occipital regions (Figure 1). However, neither global $\left(12.1 \mu \mathrm{V}^{2} / \mathrm{Hz} \pm 5.4\right.$ vs. $\left.13.9 \mu \mathrm{V}^{2} / \mathrm{Hz} \pm 5.3, \mathrm{p}=0.44\right)$ nor topographic differences were observed for MDD men relative to age-matched male controls (Figure 1).

Given the sex-related differences in all-night global SWA observed, analyses both unstratified and stratified by sex were performed to examine the role of NREM period. In the unstratified analysis, a 2 (group) by 3 (NREM period) mixed model ANOVA was utilized, which showed a main effect of NREM period $\left(\mathrm{F}_{2,46}=39.28, \mathrm{p}<0.0001\right)$ and a significant group $\mathrm{x}$ period interaction $\left(\mathrm{F}_{2,92}=4.83, \mathrm{p}=0.01\right)$, without main effect of group $\left(\mathrm{F}_{1,46}=0.81 ; \mathrm{p}=0.37\right)$. Post hoc analyses, using Tukey's HSD to correct for multiple comparisons, showed a significant decline of global SWA from NREM1 to NREM3 for both MDD $(p=0.0001)$ and HC $(\mathrm{p}=0.001)$, with no significant differences between MDD and $\mathrm{HC}$ groups in any NREM period (Figure 2). In the stratified analyses, a 4 (group) by 3 (NREM period) mixed model ANOVA was utilized to examine global SWA across the night among groups stratified by sex. ANOVA demonstrated a main effect of period $\left(\mathrm{F}_{2,44}=40.06, \mathrm{p}<0.0001\right)$ and a group $\mathrm{x}$ period interaction $\left(\mathrm{F}_{6,88}=5.76, \mathrm{p}<0.0001\right)$. Post hoc analysis demonstrated a significant decline of global SWA from
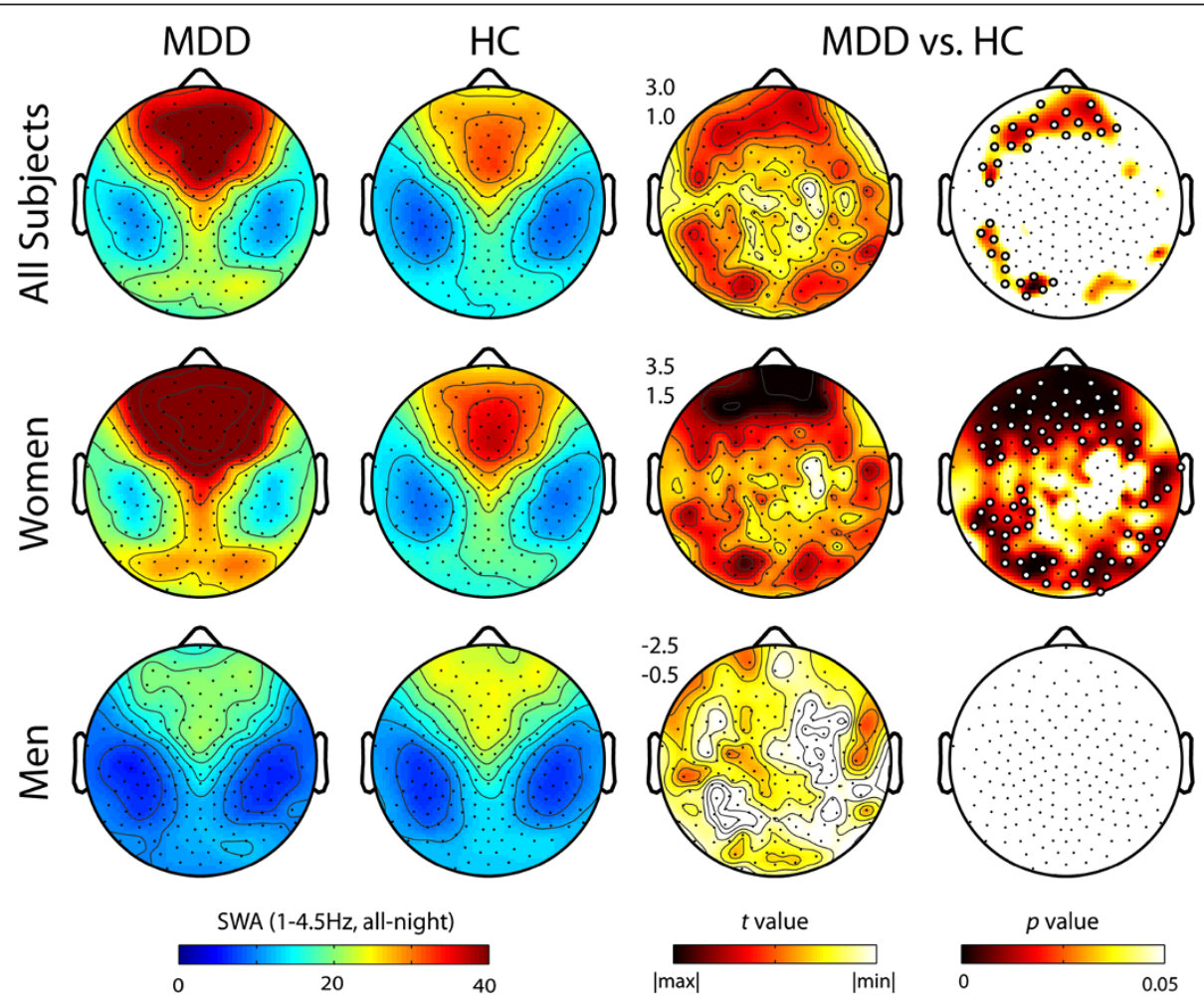

Figure 1 Topographic all-night SWA (1-4.5 Hz) in MDD subjects versus healthy controls, both unstratified and stratified by sex. T-values plotted for the comparisons between groups (2-tailed, unpaired t-test) at each channel. The minimum and maximum t-values for each map are plotted in white and black respectively, with the corresponding numeric range for color scale (upper left). Corresponding p-values plotted for each channel with white dots denoting channels with significant between-group differences following statistical non-parametric mapping with suprathreshold cluster tests to correct for multiple comparisons. 


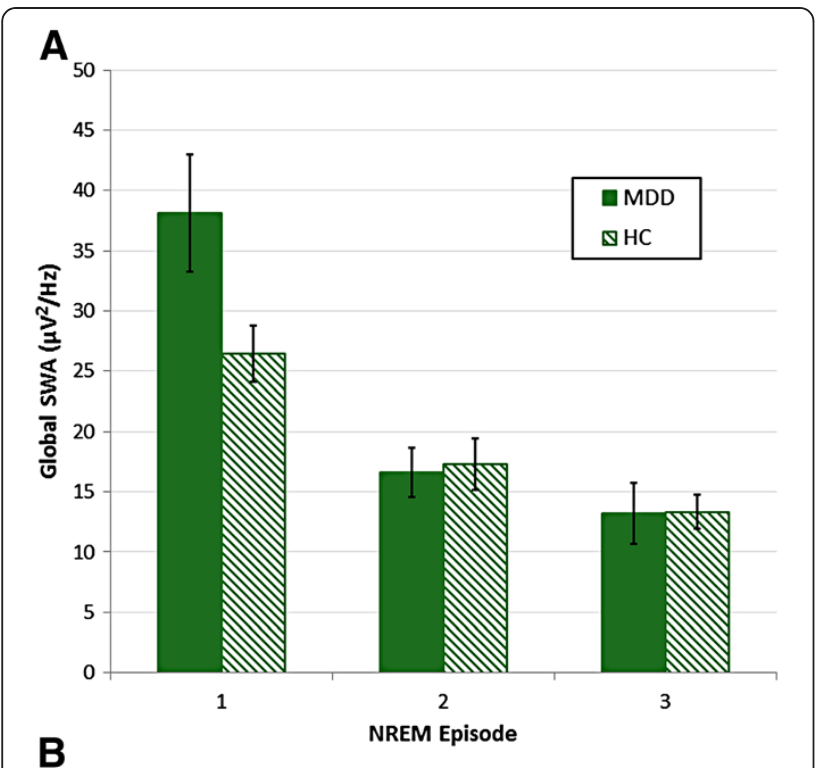

B

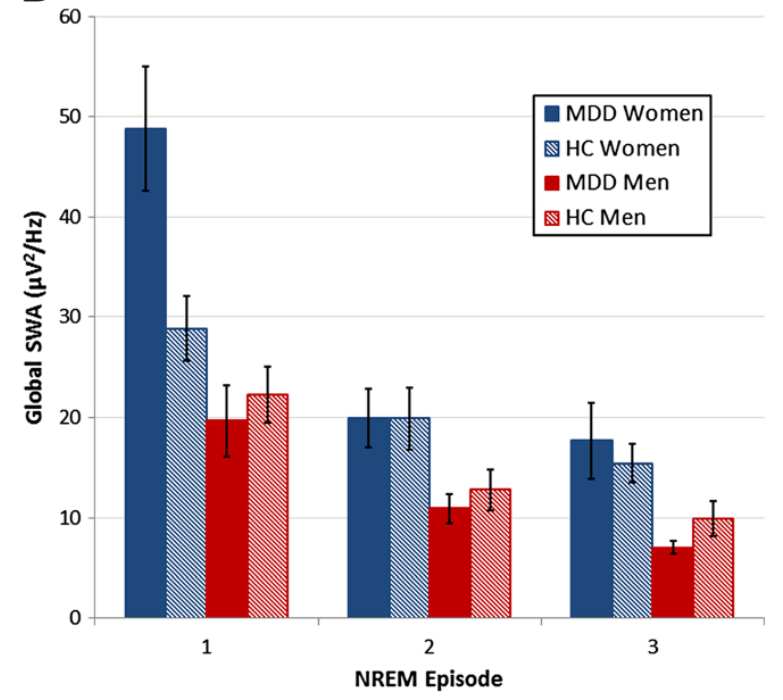

Figure 2 Global SWA (1-4.5Hz) across NREM periods for MDD and healthy controls $A$ ) unstratified and B) stratified by sex. Error bars represent standard error of the mean.

NREM1 to NREM3 for MDD $(\mathrm{p}=0.0001)$ and HC women $(p=0.012)$, but not for MDD or HC men after correcting for multiple comparisons (Figure 2). MDD women showed a trend towards greater global SWA than age-matched $\mathrm{HC}$ women $(\mathrm{p}=0.097)$ in NREM1, however, there were no other differences between MDD subjects of either sex and age-matched $\mathrm{HC}$ subjects in NREM2 or NREM3.

Subsequent topographic analysis of SWA in NREM1 demonstrated nearly identical patterns as all-night SWA in both stratified and unstratified analyses (See Additional file 1: Figure S1). Additional exploratory topographic analyses of SWA in NREM2 and NREM3 demonstrated no significant differences between groups (either stratified or unstratified by sex).
Fitted exponential decay functions are depicted in Figure 3 and calculated SWA time course variables presented in Table 2. In the unstratified analysis, there was a trend for greater $\mathrm{SWA}_{0}$ in MDD relative to control subjects $(\mathrm{p}=0.10)$. When SWA time course was stratified by sex, MDD women demonstrated significantly greater $\mathrm{SWA}_{0}$ relative to $\mathrm{HC}$ women $(\mathrm{p}=0.03)$. There were no other significant differences in SWA time course variables in either unstratified or stratified analyses.

Although MDD and HC subjects were age and sexmatched in the unstratified analysis, when stratified by sex, the ages between male and female participants were not identical (Table 1). Therefore, a secondary analysis was performed using analysis of covariance (ANCOVA) to compare differences in all-night global SWA among all groups that included age as a covariate. ANCOVA revealed a significant main effect of sex $\left(F_{1,55}=14.00\right.$, $\mathrm{p}=0.0004$ ), a trend towards main effect of diagnosis $\left(F_{1,55}=3.24, p=0.077\right)$, a significant diagnosis $x$ sex interaction $\left(\mathrm{F}_{1,55}=5.98, \mathrm{p}=0.018\right)$, and a significant effect of age $\left(\mathrm{F}_{1,55}=4.99, \mathrm{p}=0.030\right)$. Adjusted means for SWA were marginally different compared to unadjusted values (adjusted mean \pm std error: MDD women: $28.05 \pm 1.94$, MDD men: $13.56 \pm 2.60$, HC women: $18.55 \pm 1.95$, HC men: $15.00 \pm 2.56 \mu \mathrm{V}^{2} / \mathrm{Hz}$ ). Post hoc analysis showed MDD women demonstrated significantly greater SWA than HC women $(\mathrm{p}=0.0009)$, HC men $(\mathrm{p}=0.0002)$, and MDD men $(p<0.0001)$. However, there were no other significant differences in SWA between groups including between $\mathrm{HC}$ women and $\mathrm{HC}$ men $(\mathrm{p}=0.28)$.

There were no significant correlations of SWA (either all-night or in NREM1) with HRSD scores in MDD, either stratified or unstratified by sex. Additionally, there were no significant correlations of polysomnographic variables with global or topographic SWA (either allnight or NREM1) in HC or MDD subjects, either stratified or unstratified by sex.

\section{Discussion}

Our findings demonstrated an increase in SWA in young to middle aged women with unipolar MDD. Moreover, increases in SWA in MDD women primarily occurred during the first portion of the night and were most prominent in prefrontal regions. In contrast, men with MDD did not demonstrate significant changes in SWA, corroborating prior investigations that demonstrate sexspecific differences in SWA in MDD $[29,30]$. Strengths of this investigation include age and sex-matching of subjects, which allowed for analyses stratified by sex, as well as lack of confounding psychotropic medications and use of hdEEG for spectral analysis. These results highlight the importance of both sex and EEG topography in the evaluation of SWA in mood disorders. 


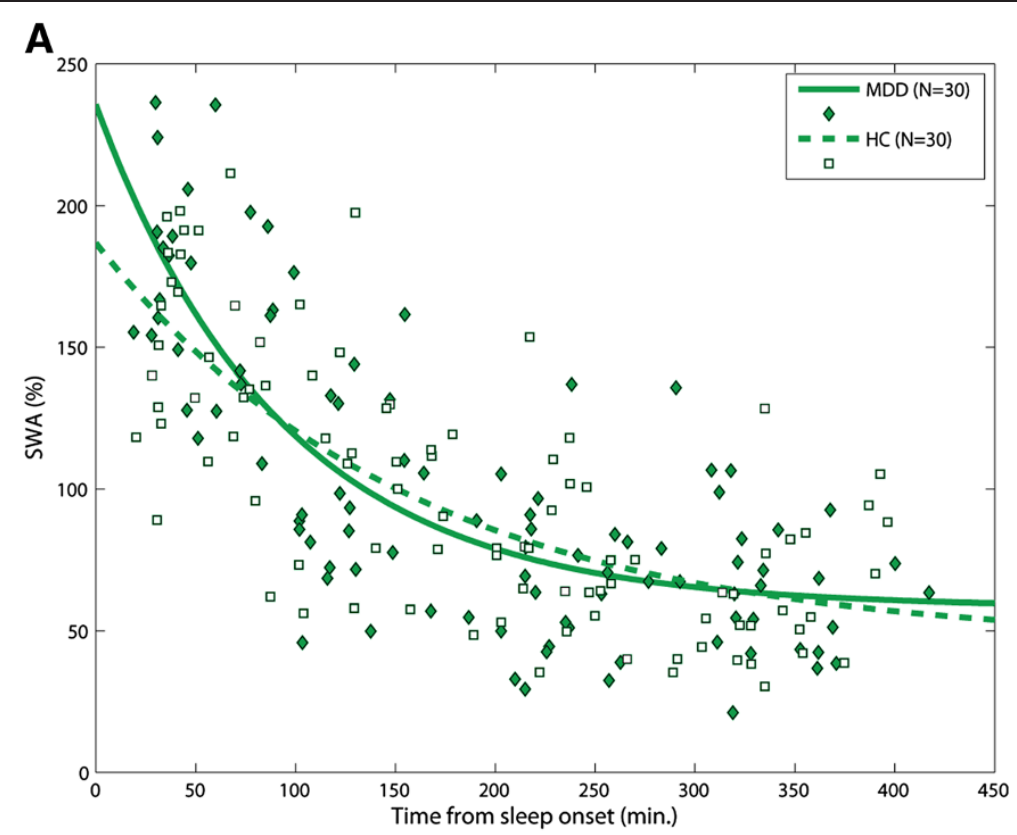

B

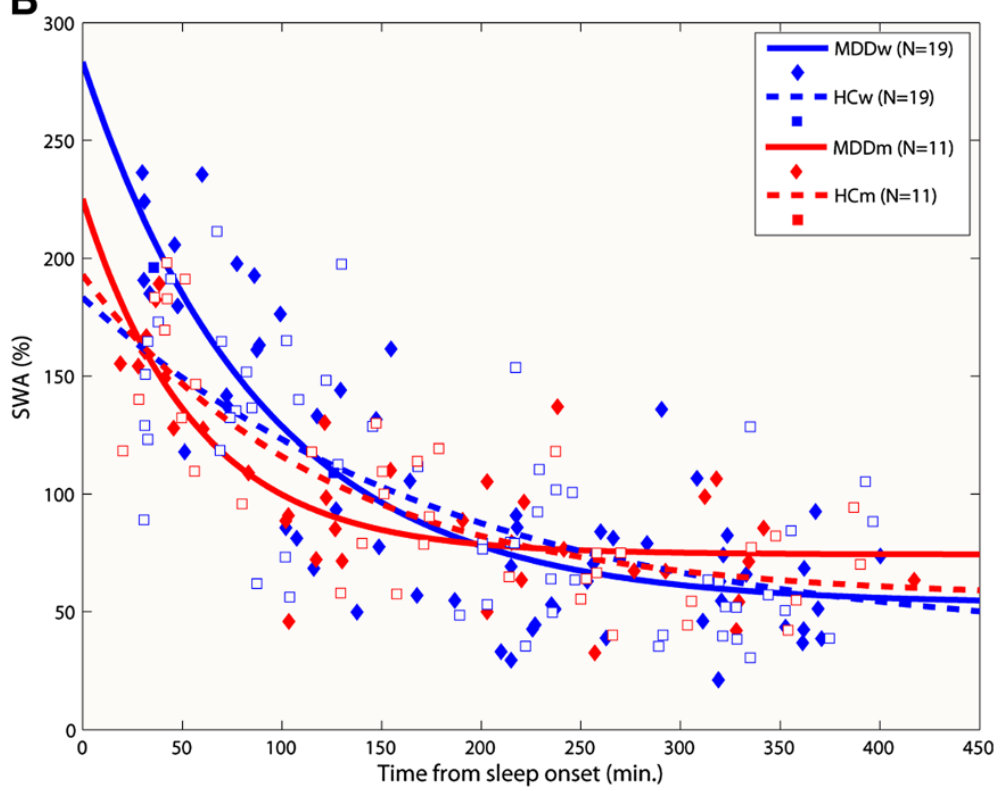

Figure 3 Time course of relative slow wave activity (SWA) across non-rapid eye movement (NREM) sleep periods. Individual SWA values per NREM sleep period (expressed as a percentage of all night SWA) are plotted at period midpoint relative to sleep onset for MDD and healthy controls $\mathbf{A}$ ) unstratified and $\mathbf{B}$ ) stratified by sex. All data from the first four NREM periods were included to maximize fit of the exponential function. Lines represent exponential functions that were fitted to the data using the equation $S W A_{t}=\left(S W A_{0} * e^{-r t}\right)+S W A_{\infty}$, in which SWA $A_{t}$ is the SWA value at a given time point $t, S W A_{0}$ represents the hypothetical SWA value at time $0, t$ indicates time (min) from sleep onset, $r$ is the rate $\left(\mathrm{min}^{-1}\right)$ of exponential decay, and SWA $\mathrm{A}_{\infty}$ indicates the SWA value of the decay function's asymptote.

The findings of this study must be taken in context of the ratio of women to men, which was roughly $2: 1$, approximating the differential prevalence of MDD by sex in the general population [46]. Notably, two known prior studies in the literature that have demonstrated similarly increased SWA or delta counts (using period amplitude analysis) in depressive illness relative to controls have both had disproportionately high ratios of female to male MDD subjects of greater than 3:1; however, neither study stratified their analyses by sex $[47,48]$. Our stratified analysis found that in women with MDD, SWA was globally increased relative to female $\mathrm{HC}$, but was most prominently increased in bilateral prefrontal cortical regions, consistent with multiple 
Table 2 SWA time course variables derived from fitted exponential decay functions*

\begin{tabular}{|c|c|c|c|c|c|c|c|c|c|}
\hline & MDD & $\mathrm{HC}$ & & MDD Females & HC Females & & MDD Males & HC Males & \\
\hline & $(N=30)$ & $(N=30)$ & $p$ & $(N=19)$ & $(N=19)$ & $p$ & $(N=11)$ & $(N=11)$ & $p$ \\
\hline $\mathrm{R}^{2}$ & 0.63 & 0.55 & - & 0.71 & 0.51 & - & 0.66 & 0.65 & - \\
\hline$S W A_{0}$ & 177.34 & 141.00 & 0.10 & 230.04 & 146.86 & 0.03 & 150.79 & 137.30 & 0.68 \\
\hline$(\mathrm{Cl})$ & $(143.41-211.27)$ & $(113.44-168.55)$ & & $(176.43-283.66)$ & $(95.57-198.15)$ & & $(89.50-212.09)$ & $(102.63-171.97)$ & \\
\hline$r$ & 0.0108 & 0.0063 & 0.18 & 0.0112 & 0.0053 & 0.15 & 0.0178 & 0.0082 & 0.16 \\
\hline$(\mathrm{Cl})$ & $(0.0060-0.155)$ & $(0.0017-0.0110)$ & & $(0.0060-0.0163)$ & $(-0.0011-0.0116)$ & & $(0.0057-0.0299)$ & $(0.0011-0.0154)$ & \\
\hline$S W A_{\infty}$ & 58.30 & 45.82 & 0.52 & 53.24 & 36.49 & 0.63 & 74.35 & 55.74 & 0.28 \\
\hline$(\mathrm{Cl})$ & $(41.86-74.74)$ & $(10.23-81.40)$ & & $(33.41-73.08)$ & $(-33.48-106.47)$ & & $(60.13-88.56)$ & $(22.47-89.02)$ & \\
\hline
\end{tabular}

*Values derived from exponential functions that were fitted to the data using the equation $S_{W A}=\left(S W A_{0} * e^{-r t}\right)+S W A_{\infty}$ in which SWA is the $S W A$ value at a given time point $t, S W A_{0}$ represents the hypothetical SWA value at time $0, t$ indicates time (min) from sleep onset, $r$ is the rate (min ${ }^{-1}$ ) of exponential decay, and $S W A_{\infty}$ indicates the SWA value of the decay function's asymptote. $\mathrm{Cl}$ denotes the $95 \%$ confidence interval. $R^{2}$ for the exponential function reflects goodness of fit.

structural and functional neuroimaging investigations which have demonstrated the importance of the prefrontal cortex in the neurobiology of depression $[49,50]$. However, it is currently not clear how increased SWA in women with MDD is related to the pathophysiology of the disorder.

This study highlights the importance of electrode placement in the evaluation of SWA during sleep in mood disorders. Notably, we did not find a significant difference in SWA between MDD and control subjects in central channels, including analyses stratified by sex. Since the previous scientific literature regarding SWA in depression has utilized central EEG derivations, it is conceivable that the aforementioned inconsistencies between previous studies may be in part due to a lack of topographical resolution or increased variability of SWA in central regions. Thus, the findings of this study suggest spectral analysis of sleep using central derivations may be inadequate to capture the alterations in SWA occurring across the cortex in mood disorders.

There are limitations of this study that merit discussion. Although a prior investigation has demonstrated decreases in SWA in men with MDD [30], we did not find statistically significant differences in men with MDD relative to matched $\mathrm{HC}$ subjects. However, it is possible that our study was underpowered to detect differences in SWA or decline of SWA across sleep among males. It is also possible that significant prefrontal increases in SWA in MDD in the unstratified analysis would not have been demonstrated had the ratio of men to women been proportionate. Furthermore, subjects did not have an adaptation night in the sleep laboratory, which may have affected results, if groups were differentially affected by first-night effects. Although delta EEG activity is not significantly different in the luteal versus follicular phase of the menstrual cycle in either healthy subjects or patients with severe premenstrual syndrome, it is also possible that not assessing menstrual status at the time of polysomnography may have influenced the results [51]. Finally, subjects were young to middle aged, and thus, results may not be applicable to older subjects with MDD, as it has been suggested alterations in SWA in MDD are more apparent in young to middle aged cohorts [31].

This study was not able to determine whether increased SWA in MDD is a causal phenomenon for the disorder or an epiphenomenon resulting from chronic sleep disruption associated with the illness. Topographic increases in SWA in depressed women observed in this study are not specific to MDD, as such findings are also seen during recovery sleep following acute sleep deprivation [33-35]. It is intriguing that increases in SWA are observed in both MDD and sleep deprivation paradigms, as both are associated with impairments in cognitive functions such as attention and working memory, as well as subjective fatigue [52,53]. Given the results of this study, further experiments are warranted that examine the relationship between sleep homeostatic processes and executive function in MDD, using sleep restriction and/or sleep delay paradigms [28], and the effects of sex on these parameters.

Our results suggest that increased SWA, particularly during early portions of the night, may be related to the pathophysiology of MDD in women. In accordance with this hypothesis, it has been previously proposed that increases in NREM sleep intensity may be depressogenic [54], and a recent demonstration that selective slowwave deprivation has an acute antidepressant response supports this contention [55]. In addition, when compared to healthy controls, young MDD women exhibited greater low-frequency EEG activity in frontal regions during extended wakefulness [56], which has been demonstrated to be a waking correlate of sleep homeostasis $[34,57]$. Both increased SWA during baseline sleep and an enhanced homeostatic response to sleep deprivation paradigms have also been reported, suggesting that MDD women live with an elevated level of homeostatic sleep pressure [31,58,59]. Although our results would support this contention, our experimental 
design did not include manipulations of sleep homeostasis, and thus only provides partial evidence for this hypothesis. Moreover, given our cross-sectional design, we were not able to determine whether increased SWA in MDD is a state or trait marker for MDD in women, nor are we able to determine if increased SWA is causative for depressive symptoms or an epiphenomenon of some other neurobiological process, which may be mediated by sex-related differences in neurobiology.

The differences in brain structure and function between men and women, and how these alterations may relate to the pathophysiology of mood disorders is a complex topic, as dissecting which components are biologically determined and which are the consequence of environmental conditioning due to gender-related sociocultural experience is fraught with difficulty [60]. Still, there is significant evidence that there are sex-related differences in gene expression, epigenetic regulatory mechanisms, hypothalamuspituitary-adrenal axis regulation, and modulation of neurotransmitter systems by neurosteroids between men and women, which may relate to differences in susceptibility to neuropsychiatric illness [61-63]. It is currently not clear how our neurophysiologic finding of increased SWA in women with MDD may be related to any of these mechanisms, and thus, further research that examines SWA in relation to sex-dependent hormonal, genetic, and epigenetic mechanisms in mood disorders may provide deeper insights into this conceptual framework.

\section{Conclusions}

In conclusion, sleep disturbance and MDD are intimately linked; however, previous research that has examined the role of SWA in depression has been inconsistent. This study demonstrates sex-related differences in SWA, in which women with MDD had elevated SWA, most prominently in prefrontal regions and during the first portion of the night. Failure to account for sex-related differences in SWA and the use of limited EEG derivations to perform spectral analysis in prior investigations may have contributed to inconsistencies in the literature. However, given the heterogeneity of MDD $[50,64]$, it is also plausible that other factors contribute to alterations in SWA in the disorder. Further research is indicated to examine the effects of sex-specific neurobiology, neurocognitive symptoms, and SWA in mood disorders to clarify how increased SWA in women with MDD is related to the pathophysiology of the disorder.

\section{Additional file}

Additional file 1: Figure S1. Topographic SWA (1-4.5 Hz) during NREM1 in MDD subjects versus healthy controls, both unstratified and stratified by sex. T-values plotted for the comparisons between groups (2-tailed, unpaired $t$-test) at each channel. The minimum and maximum $\mathrm{t}$-values for each map are plotted in white and black respectively, with the corresponding numeric range for color scale (upper left). Corresponding p-values plotted for each channel with white dots denoting channels with significant between-group differences following statistical nonparametric mapping with suprathreshold cluster tests to correct for multiple comparisons.

\section{Competing interests}

DTP has owned stock in Pfizer, and has received honoraria from Oakstone Medical Publishing and royalties from Cambridge University Press. MJP has received unrelated research support from Sanofi-Aventis. GT has consulted for Sanofi-Aventis and Takeda, and he is currently the David P. White Chair in Sleep Medicine at the University of Wisconsin-Madison, endowed by Phillips Respironics. GT has also received unrelated research support from Phillips Respironics. RMB has consulted for Merck and Sanofi-Aventis. All other authors declare they have no competing interests.

\section{Authors' contributions}

DTP designed the study, managed literature searches and analyses, and wrote the initial and final draft of the manuscript. ECL and MJP contributed to the study design, evaluation of participants, management of hdEEG processing and analyses, and writing the article. MRG, TW, and JJG performed statistical analyses and hdEEG processing, designed tables/figures, and contributed to the writing of the manuscript. BA performed data analysis and interpretation, and also contributed to the writing of the manuscript. GT contributed to the study design, data analysis and interpretation, and to writing the article. RMB contributed by being the principal investigator, contributing to the study design, participating in data analysis and interpretation, and writing the article. All authors contributed to and have approved the final manuscript.

\section{Acknowledgements}

This research was funded by the National Institute of Mental Health (5P2OMH077967 to GT and RMB, and F30MH082601 to ECL) and the National Alliance for Research on Schizophrenia and Depression Young Investigator Award to MJP. The NIMH and NARSAD had no further role in the study design, data collection, analysis and interpretation of the data, and the decision to submit the paper for publication.

Received: 22 November 2011 Accepted: 10 September 2012 Published: 18 September 2012

\section{References}

1. American Psychiatric Association: Diagnostic and Statistical Manual of Mental Disorders: Fourth Edition, Text Revision ed. Washington, D.C.: American Psychiatric Association; 2000.

2. Nutt D, Wilson S, Paterson L: Sleep disorders as core symptoms of depression. Dialogues Clin Neurosci 2008, 10(3):329-336.

3. Breslau N, Roth T, Rosenthal L, Andreski P: Sleep disturbance and psychiatric disorders: a longitudinal epidemiological study of young adults. Biol Psychiatry 1996, 39(6):411-418.

4. Chang PP, Ford DE, Mead LA, Cooper-Patrick L, Klag MJ: Insomnia in young men and subsequent depression. The Johns Hopkins Precursors Study. Am J Epidemiol 1997, 146(2):105-114.

5. Roberts RE, Shema SJ, Kaplan GA, Strawbridge WJ: Sleep complaints and depression in an aging cohort: A prospective perspective. Am J Psychiatry 2000, 157(1):81-88

6. Buysse DJ, Angst J, Gamma A, Ajdacic V, Eich D, Rossler W: Prevalence, course, and comorbidity of insomnia and depression in young adults. Sleep 2008, 31(4):473-480.

7. Szklo-Coxe M, Young T, Peppard PE, Finn LA, Benca RM: Prospective associations of insomnia markers and symptoms with depression. Am J Epidemiol 2010, 171(6):709-720.

8. Wojnar M, Ilgen MA, Wojnar J, McCammon RJ, Valenstein M, Brower KJ: Sleep problems and suicidality in the National Comorbidity Survey Replication. J Psychiatr Res 2009, 43(5):526-531.

9. Goldstein TR, Bridge JA, Brent DA: Sleep disturbance preceding completed suicide in adolescents. J Consult Clin Psychol 2008, 76(1):84-91.

10. Dombrovski AY, Mulsant BH, Houck PR, Mazumdar S, Lenze EJ, Andreescu C, Cyranowski JM, Reynolds CF 3rd: Residual symptoms and recurrence 
during maintenance treatment of late-life depression. J Affect Disord 2007, 103(1-3):77-82.

11. Karp JF, Buysse DJ, Houck PR, Cherry C, Kupfer DJ, Frank E: Relationship of variability in residual symptoms with recurrence of major depressive disorder during maintenance treatment. Am J Psychiatry 2004, 161(10):1877-1884.

12. Paykel ES, Ramana R, Cooper Z, Hayhurst H, Kerr J, Barocka A: Residual symptoms after partial remission: an important outcome in depression. Psychol Med 1995, 25(6):1171-1180.

13. Benca RM, Obermeyer WH, Thisted RA, Gillin JC: Sleep and psychiatric disorders. A meta-analysis. Arch Gen Psychiatry 1992, 49(8):651-668.

14. Steiger A, Kimura M: Wake and sleep EEG provide biomarkers in depression. J Psychiatr Res 2010, 44(4):242-252.

15. Rechtschaffen A, Kales A: A Manual of Standardized Terminology Techniques and Scoring System for Sleep States of Human Subjects. University of California at Los Angeles: Brain information Service/Brain Research institute; 1968.

16. Iber C, Ancoli-Israel S, Chesson AL, Quan SF, American Academy of Sleep Medicine: The AASM Manual for the Scoring of Sleep and Associated Events: Rules, Terminology, and Technical Specifications. First editionth edition. Westchester, Illinois: American Academy of Sleep Medicine; 2007.

17. Borbely AA, Baumann F, Brandeis D, Strauch I, Lehmann D: Sleep deprivation: effect on sleep stages and EEG power density in man. Electroencephalogr Clin Neurophysiol 1981, 51(5):483-495.

18. Dijk DJ: Regulation and functional correlates of slow wave sleep. J Clin Sleep Med 2009, 5 (2 Suppl):S6-S15.

19. Borbely AA, Wirz-Justice A: Sleep, sleep deprivation and depression. A hypothesis derived from a model of sleep regulation. Hum Neurobiol 1982, 1(3):205-210

20. Achermann P, Dijk DJ, Brunner DP, Borbely AA: A model of human sleep homeostasis based on EEG slow-wave activity: quantitative comparison of data and simulations. Brain Res Bull 1993, 31(1-2):97-113.

21. Borbely AA: A two process model of sleep regulation. Hum Neurobio 1982, 1(3):195-204.

22. Borbely AA: The S-deficiency hypothesis of depression and the two-process model of sleep regulation. Pharmacopsychiatry 1987, 20(1):23-29.

23. Borbely AA, Tobler I, Loepfe M, Kupfer DJ, Ulrich RF, Grochocinski V, Doman J, Matthews G: All-night spectral analysis of the sleep EEG in untreated depressives and normal controls. Psychiatry Res 1984, 12(1):27-33.

24. Hoffmann R, Hendrickse W, Rush AJ, Armitage R: Slow-wave activity during non-REM sleep in men with schizophrenia and major depressive disorders. Psychiatry Res 2000, 95(3):215-225

25. Mendelson WB, Sack DA, James SP, Martin JV, Wagner R, Garnett D, Milton J, Wehr TA: Frequency analysis of the sleep EEG in depression. Psychiatry Res 1987, 21(2):89-94.

26. Armitage R, Calhoun JS, Rush AJ, Roffwarg HP: Comparison of the delta EEG in the first and second non-REM periods in depressed adults and normal controls. Psychiatry Res 1992, 41(1):65-72.

27. Landolt HP, Gillin JC: Similar sleep EEG topography in middle-aged depressed patients and healthy controls. Sleep 2005, 28(2):239-247.

28. Brower KJ, Hoffmann R, Conroy DA, Arnedt JT, Armitage R: Sleep homeostasis in alcohol-dependent, depressed and healthy control men. Eur Arch Psychiatry Clin Neurosci 2011, 261(8):559-566.

29. Armitage R, Hoffmann R, Fitch T, Trivedi M, Rush AJ: Temporal characteristics of delta activity during NREM sleep in depressed outpatients and healthy adults: group and sex effects. Sleep 2000, 23(5):607-617.

30. Armitage R, Hoffmann R, Trivedi M, Rush AJ: Slow-wave activity in NREM sleep: sex and age effects in depressed outpatients and healthy controls. Psychiatry Res 2000, 95(3):201-213.

31. Armitage R: Sleep and circadian rhythms in mood disorders. Acta Psychiatr Scand Suppl 2007, 433:104-115.

32. Werth $E$, Achermann $P$, Borbely AA: Fronto-occipital EEG power gradients in human sleep. J Sleep Res 1997, 6(2):102-112.

33. Cajochen C, Foy R, Dijk DJ: Frontal predominance of a relative increase in sleep delta and theta EEG activity after sleep loss in humans. Sleep Res Online 1999, 2(3):65-69.

34. Finelli LA, Baumann $H$, Borbely AA, Achermann P: Dual electroencephalogram markers of human sleep homeostasis: correlation between theta activity in waking and slow-wave activity in sleep. Neuroscience 2000, 101(3):523-529.

35. Tinguely G, Finelli LA, Landolt HP, Borbely AA, Achermann P: Functional EEG topography in sleep and waking: state-dependent and stateindependent features. Neurolmage 2006, 32(1):283-292.
36. First M, Spitzer R, Gibbon M, Williams J: Structured clinical interview for DSMIV-TR axis I disorders, research version, patient edition. New York: Biometrics Research, New York State Psychiatric Institute; 2002

37. Hamilton M: A rating scale for depression. J Neurol Neurosurg Psychiatry 1960, 23:56-62.

38. First M, Spitzer R, Gibbon M, Williams J: Structured clinical interview for DSMIV-TR axis I disorders, research version, non-patient edition (SCID-I/NP). New York: Biometrics Research, New York State Psychiatric Institute; 2002.

39. Goncharova II, MCFarland DJ, Vaughan TM, Wolpaw JR: EMG contamination of EEG: spectral and topographical characteristics. Clin Neurophysiol 2003, 114(9):1580-1593.

40. Dijk DJ, Brunner DP, Borbély AA: Time course of EEG power density during long sleep in humans. Am J Physiol 1990, 258(3Pt2):R650-R661.

41. Feinburg I, Floyd TC: Systematic trends across the night in human sleep cycles. Psychophysiology 1979, 16(3):283-291.

42. Mourtazaev MS, Kemp B, Zwinderman AH, Kamphuisen HA: Age and gender affect different characteristics of slow waves in the sleep EEG. Sleep 1995, 18(7):557-564.

43. Ehlers CL, Kupfer DJ: Slow-wave sleep: do young adult men and women age differently? J Sleep Res 1997, 6(3):211-215

44. Latta F, Leproult R, Tasali E, Hofmann E, Van Cauter E: Sex differences in delta and alpha EEG activities in healthy older adults. Sleep 2005, 28(12):1525-1534.

45. Nichols TE, Holmes AP: Nonparametric permutation tests for functional neuroimaging: a primer with examples. Hum Brain Mapp 2002, 15(1):1-25.

46. Kessler RC, McGonagle KA, Swartz M, Blazer DG, Nelson CB: Sex and depression in the National Comorbidity Survey. I: Lifetime prevalence, chronicity and recurrence. J Affect Disord 1993, 29(2-3):85-96.

47. Reynolds CF 3rd, Kupfer DJ, Taska LS, Hoch CH, Sewitch DE, Grochocinski VJ: Slow wave sleep in elderly depressed, demented, and healthy subjects. Sleep 1985, 8(2):155-159.

48. Schwartz PJ, Rosenthal NE, Wehr TA: Band-specific electroencephalogram and brain cooling abnormalities during NREM sleep in patients with winter depression. Biol Psychiatry 2001, 50(8):627-632.

49. Drevets WC, Price JL, Furey ML: Brain structural and functional abnormalities in mood disorders: implications for neurocircuitry models of depression. Brain Struct Funct 2008, 213(1-2):93-118.

50. Krishnan $\mathrm{V}$, Nestler EJ: Linking molecules to mood: new insight into the biology of depression. Am J Psychiatry 2010, 167(11):1305-1320.

51. Baker FC, Sassoon SA, Kahan T, Palaniappan L, Nicholas CL, Trinder J, Colrain IM: Perceived poor sleep quality in the absence of polysomnographic sleep disturbance in women with severe premenatrual syndrome. J Sleep Res, In Press.

52. Marazziti D, Consoli G, Picchetti M, Carlini M, Faravelli L: Cognitive impairment in major depression. Eur J Pharmacol 2010, 626(1):83-86.

53. Goel N, Rao H, Durmer JS, Dinges DF: Neurocognitive consequences of sleep deprivation. Semin Neurol 2009, 29(4):320-339.

54. Beersma DG, van den Hoofdakker RH: Can non-REM sleep be depressogenic? J Affect Disord 1992, 24(2):101-108.

55. Landsness EC, Goldstein MR, Peterson MJ, Tononi G, Benca RM: Antidepressant effects of selective slow wave sleep deprivation in major depression: a high-density EEG investigation. J Psychiatr Res 2011, 45(8):1019-1026.

56. Birchler-Pedross A, Frey S, Chellappa SL, Gotz T, Knoblauch V, Wirz-Justice A, Cajochen $C$ : Higher frontal EEG synchronisation in young women with major depression: a marker for increased homeostatic sleep pressure? Sleep 2011, 34(12):1699-1706.

57. Cajochen C, Wyatt JK, Czeisler CA, Dijk DJ: Separation of circadian and wake duration-dependent modulation of EEG activation during wakefulness. Neuroscience 2002, 114(4):1047-1060.

58. Birchler-Pedross A, Frey S, Chellappa SL, Götz T, Brunner P, Knoblauch V, Wirz-Justice A, Cajochen C: Higher frontal EEG synchronization in young women with major depression: a marker for increased homeostatic sleep pressure? Sleep 2011, 34(12):1699-706

59. Frey S, Birchler-Pedross A, Hofstetter M, Brunner P, Götz T, Münch M, Blatter K, Knoblauch V, Wirz-Justice A, Cajochen C: Young women with major depression live on higher homeostatic sleep pressure than healthy controls. Chronobiol Int 2012, 29(3):278-294.

60. Legato MJ: The skewed sex distribution in affective disorders-a diagnostic, social, or biological problem? Prog Brain Res 2010, 186:159-166. 
61. Qureshi IA, Mehler MF: Genetic and epigenetic underpinnings of sex differences in the brain and in neurological and psychiatric disease susceptibility. Prog Brain Res 2010, 186:77-95.

62. Reddy DS: Neurosteroids: endogenous role in the human brain and therapeutic potentials. Prog Brain Res 2010, 186:113-137.

63. Young E, Korszun A: Sex, trauma, stress hormones and depression. Mol Psychiatry 2010, 15(1):23-28.

64. Tsuno N, Besset A, Ritchie K: Sleep and depression. J Clin Psychiatry 2005, 66(10):1254-1269.

doi:10.1186/1471-244X-12-146

Cite this article as: Plante et al:: Sex-related differences in sleep slow

wave activity in major depressive disorder: a high-density EEG

investigation. BMC Psychiatry 2012 12:146.

\section{Submit your next manuscript to BioMed Central and take full advantage of:}

- Convenient online submission

- Thorough peer review

- No space constraints or color figure charges

- Immediate publication on acceptance

- Inclusion in PubMed, CAS, Scopus and Google Scholar

- Research which is freely available for redistribution 